\title{
A PROPÓSITO DE LOS ANTECEDENTES DEL PENSAMIENTO POLÍTICO CUBANO Óscar Puig Corral*
}

\section{REGARDING THE ORIGINS OF CUBAN POLITICAL THOUGHT IN THE CONSTITUTION OF GUÁIMARO (1869)}

RESUMEN: Pretender analizar hechos ocurridos hace más de 148 años es arriesgado, si no se tiene en cuenta el momento histórico concreto. El riesgo es mayor al ser los hechos en cuestión parte importante de la columna fundacional de la historia patria cubana. No solo se analizan los primeros días de la gesta independentista; aun más importante, se intenta arrojar luz sobre la necesidad de crear un cuerpo armónico con forma de ley que uniera con un objetivo común a los patriotas cubanos. Curioso proceso este que iba en contrario al de la mayoría, primero una Constitución política que fijara los derroteros insulares durante la contienda; luego, se pensaba en cómo ganar la guerra contra España. Fue la Constitución de Guáimaro, nombre que tomó del poblado dónde se llevó a cabo la Asamblea Constituyente. Hacemos especial énfasis en la influencia de la Constitución de Cádiz y en la figura ilustre del presbítero Félix Varela como primer impulsor de las ideas sobre el constitucionalismo en Cuba.

Palabras Clave: Cuba, Asamblea Constituyente, constitucionalismo, Ilustración, revolución.
ABSTRACT: Any attempt to interpret the events that happened 148 years ago can be very risky if one does not consider the historic context. This risk is even greater since these particular events are foundational to Cuban history. We will not only analyze the first days of the independence movement but more importantly, we will shed light on the need of a cohesive structure shaped by a law that unified the Cuban patriots. It was a very unusual process indeed, in that it aimed at establishing first a Constitution to regulate the Island's course during the war, and only then turning its attention to defeating Spain. This "Constitution of Guáimaro" was named after the town which brought about the Constitutional Assembly. We will emphasize the influence of the Cadiz Constitution and the celebrated priest, Felix Varela, as the first promoter of constitutional ideas in Cuba.

KEYwORDS: Cuba, Constitutional Assembly, constitutionalism, Enlightenment, revolution.

\footnotetext{
* Universidad de Oriente, Santiago de Cuba.
} 


\author{
A PROPÓSITO DE \\ LOS ANTECEDENTES \\ DEL PENSAMIENTO \\ POLÍTICO CUBANO
}

Ala

la hora de hacer recuentos, siempre buscamos palabras o frases grandilocuentes. Cuando se trata de recuentos sobre hechos históricos, que incluso forman parte de la historia y el orgullo nacional, siempre quisiéramos ser exactos, dignos, honestos. En este caso el recuento es necesario, casi imprescindible. Se trata de retroceder en el tiempo 148 años y aterrizar en la manigua insurrecta, que confusa y corajuda, se debatía entre sacudirse a España de la espalda y la perentoria necesidad de darse un orden, un mapa, una raíz que la fijara definitivamente a la historia viva de la Patria. Eso fue la Constitución de Guáimaro. Alejados de cuestionamientos doctrinales, que no harían más que ahondar sobre lo ya ahondado, nos proponemos exponer nuestras ideas sobre los proce- sos materiales que llevaron a los jefes mambises a pensar en términos constitucionalistas y republicanos. Hablaremos, además, sobre los antecedentes directos e indirectos de esas ideas, basados en pensamientos fundadores de la misma nación cubana, que constituyen glorias de nuestro pensamiento patrio. En Guáimaro, pueblo liberado en noviembre de 1868, se fijaron los derroteros políticos y militares de la Revolución insurrecta. José Martí, con su egregio verbo, escribió: "Estaba Guáimaro más que nunca hermosa. Era el pueblo señorial como familia en fiesta. Venían el Oriente, y el Centro, y las Villas al abrazo de los fundadores". ${ }^{1}$

${ }^{1}$ José Martí, Obras completas, 1975, La Habana, Editorial de Ciencias Sociales, t. 4, p. 383. 
No es para nada ocioso recordar que el incidente que acabó de convencer a los jefes insurrectos de la urgencia de aprobar una Constitución, fue la autoproclamación de Donato Mármol, en enero de 1869, como dictador supremo de la revolución, ignorando incluso a Carlos Manuel de Céspedes y aconsejado entre otros por Leopoldo Arteaga, Pío Rosado, Manuel de Jesús Peña y por su polémico primo Eduardo Mármol. Supuestamente, el motivo fue que estos sediciosos no apreciaban dotes de conducción militar ni en Céspedes ni en Francisco Vicente Aguilera para llevar adelante la naciente revolución insurrecta. Por supuesto, lo de Donato Mármol y sus seguidores no fue más que una intentona fugaz, aunque extremadamente peligrosa. Luego, en una acalorada reunión en Tacajó, Mármol depuso sus intenciones y reconoció la autoridad superior de Céspedes, con la oportuna y sabia mediación de Francisco Vicente Aguilera. En esta reunión se acordó dotar de institucionalización al proceso revolucionario, dejando para después la elección de diputados con el rigor necesario para una Asamblea Constituyente. Esta tentativa sedicionista fue la principal razón, pero también subyacía el miedo a que el campo insurrecto fuera pasto de la espuela y el machete de caudillos militares, lo cual repetiría lo que había estado ocurriendo en otras partes de América Latina.
En una sesión previa a la convención, se reunieron Céspedes como "jefe del gobierno provisional del Departamento Oriental”; Miguel Gerónimo Gutiérrez, Eduardo Machado, Antonio Lorda, Tranquilino Valdés y Arcadio García, representantes de Villaclara; Honorato del Castillo, de Sancti Spíritus; Antonio Alcalá y Jesús Rodríguez, de Holguín; y Salvador Cisneros Betancourt, Francisco Sánchez Betancourt, Ignacio Agramonte y Miguel Betancourt, del Camagüey. Además, se incluyó en la delegación camagüeyana al joven pensador habanero Antonio Zambrana. ${ }^{2}$

Los diputados del Camagüey y Las Villas fueron en extremo insistentes en marcar el rumbo de la revolución mediante un texto constitucional. Estuvieron de acuerdo los orientales, que se sentían dispuestos a redactar algo más provisional, no con carácter de Carta Magna, sino que simplemente le diera curso viable a las necesidades militares de la guerra. Además, fue la misma razón por la cual, aparte de que había mayoría de diputados villareños y camagüeyanos, Céspedes prácticamente no se opuso a ninguna de las disposiciones redactadas por Ignacio Agramonte y Antonio Zambrana, las que luego, en algunos casos al ser malentendidas y

${ }^{2}$ Rolando Rodríguez García, "La Asamblea y la Cámara de Guáimaro", en Raíces en el tiempo, 2009, La Habana, Editorial de Ciencias Sociales, p. 101. 
en otros, por ser aplicadas demasiado al pie de la letra, pusieron en riesgo la continuidad y el éxito de la revolución independentista. Sobre la actitud de Céspedes, Martí escribió: "Céspedes se plegó a la forma (republicana) del Centro. No la creía conveniente; pero creía inconveniente las disensiones. Sacrificaba su amor propio; 10 que nadie sacrifica". ${ }^{3}$

Resumiendo los resultados de la asamblea de Guáimaro, el investigador Rolando Rodríguez expone:

La asamblea logró un objetivo esencial en la revolución que la vuelve imperecedera: estableció la unificación de los grupos insurgentes, lo que permitiría dar un paso rotundo hacia la formación de la nación y de la república cubanas; pero a decir verdad, no obtuvo su unidad en las concepciones organizativas y métodos para enfrentar la lucha. En definitiva, en ella nadie convenció a nadie. Aparte de aquella unificación de los grupos; de un lado, los resultados fueron los que impuso la mayoría que formaban los camagüeyanos secundados por los villareños, $\mathrm{y}$, de otra, las cesiones que hizo el iniciador de la gesta en aras de la revolución. ${ }^{4}$

Ahora bien, ¿cómo entender que en la mente de aquellos hombres podía operar una idea que consideraban

${ }^{3}$ Martí, op. cit., t. 22, p. 235.

${ }^{4}$ Rodríguez García, op. cit., p. 103. de mayor importancia que incluso muchas decisiones militares de suma trascendencia que debían ser tomadas con urgencia, es decir, reunirse los representantes del pueblo de Cuba en armas y dotarse de una constitución? En esta interrogante encontramos el primer botón de muestra de lo novedoso de este proceso, porque la historia de los movimientos revolucionarios y de las luchas por la independencia en América demuestra que primero se ganaba la guerra y luego se hacía la Constitución. Ahí están para probarlo los hechos de la Guerra de Independencia Norteamericana y los movimientos independentistas de América Latina. En estos procesos hubo muchas declaraciones, muchos bandos militares, muchas proclamaciones, pero constitución en el sentido propio del término, solo después de haber tomado el poder político, una vez derrotado el enemigo de la metrópoli colonial.

De modo que no deja de ser paradójico que los mambises cubanos hayan decidido adoptar formas institucionales con el nombre de constitución, en momentos en que no era ni por asomo debido ni exigido para movimiento de independencia alguno. Pero lo que más asombro causa es que se mantuvieron fieles a su constitución y trataron de cumplir su mandato, en algunos casos demasiado al pie de la letra. Salvador Cisneros Betancourt, marqués de Santa 
Lucía, movido por la admiración y el fervor hacia el espíritu constitucional y el ideal republicano, llegó a decir que prefería perder la guerra antes que perder el respeto por la constitución. En cierta forma, esta idea del marqués prefigura lo que posteriormente escribió José Martí, que trataré de citar lo más fielmente posible: "el despotismo que podría sobrevenir en caso de una guerra de independencia de baja raíz y temibles fines y que tuviera como resultado la entronización en Cuba de una dictadura militar, sería peor que el despotismo político español", es decir, que el despotismo militar cubano sería peor que el despotismo de la metrópoli, y explica Martí sus razones diciendo que sería más difícil desarraigar la dictadura militar, estando en ese futuro hipotético legitimada por el éxito y la gloria alcanzados en el campo de batalla por esos caudillos militares a los que temió y combatió toda su vida. Martí escribió esto llevado de la mano por sus más profundas convicciones republicanas, y creo que sería conveniente estudiar sus ideas en torno a estos temas, sobre todo si caemos en cuenta de que es una de las facetas que menos se lee dentro de la abundante obra martiana. Céspedes escribió en su proclama:

No nos extravían rencores, no nos halagan ambiciones, solo queremos ser libres e iguales como hizo el Creador a todos los hombres. Nosotros consagramos estos dos venerables principios: nosotros creemos que todos los hombres somos iguales: amamos la tolerancia, el orden y la justicia en todas las materias; respetamos las vidas y propiedades de todos los ciudadanos pacíficos, aunque sean los mismos españoles, residentes en este territorio; admiramos el sufragio universal, que asegura la soberanía del pueblo; deseamos la emancipación, gradual y bajo indemnización, de la esclavitud; el libre cambio con las naciones amigas que usan de reciprocidad, la representación nacional para decretar las leyes e impuestos, y en general, demandamos la religiosa observancia de los derechos imprescriptibles del hombre, constituyéndonos en nación independiente, porque así cumple a la grandeza de nuestros futuros destinos, y porque es más seguros que bajo el cetro de España nunca gozaremos del franco ejercicio de nuestros derechos. ${ }^{5}$

Entonces, para entender de alguna manera los motivos y las razones que llevaron a los insurrectos cubanos a dotarse de una constitución a escasísimos meses del Grito de Yara, debemos hablar, aunque sea en forma

${ }^{5}$ Luis Miguel García Mora y Alejandro García Álvarez (coords.), "Manifiesto de la Junta Revolucionaria de la Isla de Cuba", en Textos clásicos de la historia de Cuba, 1999, Madrid, Fundación MAPFRE, p. 24. 
abreviada, de algunos hechos que contribuyeron a formar ese sentimiento republicano, constitucionalista y liberal, en el sentido que tenía en el siglo XIX y no en la actualidad, pues en aquellos años liberal era todo aquel que era amante y defensor apasionado de la libertad, mientras que, desafortunadamente, en la actualidad se usa para designar a una corriente de pensamiento económico, más que político. Y si se quiere una prueba, acudamos a Mariana Grajales, esa leona madre de leones, cuando delante de sus hijos y con el crucifijo en la mano ordenó: "de rodillas todos, padres e hijos, delante de Cristo, que fue el primer hombre liberal que vino al mundo". ${ }^{6}$ Volviendo a los hechos que condicionaron el afán constitucionalista mambí, podríamos situarlos en los primeros años del siglo XIX, cuando en Cuba ciertas personalidades comenzaban a promover, en muchos casos intencionadamente, un proceso de alguna manera similar al que se dio en Francia con la Ilustración, evidentemente a escala mucho menor, en un país alejado de la metrópoli occidental, pero tan rico que contribuyó en el siglo XIX con la nada despreciable cifra de entre el 65 y el $70 \%$ de los ingresos del Imperio español.

\footnotetext{
${ }^{6}$ Véase sobre este famoso juramento la Carta de María Cabrales dirigida a Francisco de Paula Coronado, fechada el 6 de mayo de 1897, en Papeles de Maceo, t. II, 1998, La Habana, Editorial de Ciencias Sociales, pp. 73-75.
}

En otras palabras, en ese siglo, España vivió de las riquezas de Cuba. Muchos cubanos se dieron cuenta de que las riquezas de la isla no debían ser para los peninsulares, sino para que las disfrutaran los cubanos.

La consecuencia fue que, hacia 1810, empezó a apreciarse en Cuba un fuerte movimiento de ideas que apuntaban a una reforma educativa, así como a un conjunto de medidas políticas que se expresaron en los primeros proyectos constitucionales elaborados en Cuba, impulsados también por los acontecimientos contemporáneos en España, que dieron lugar en 1812 a la promulgación de la primera constitución ${ }^{7}$ de España y también la primera de Cuba, pues fue el primer texto constitucional vigente en la isla. Esta constitución, con todas las limitaciones del caso, del poder de la monarquía española y de la religión católica, reconocía una amplia gama de derechos civiles y políticos, heredados por supuesto de las declaraciones francesa y norteamericana.

Esa época coincide con los dos años de vigencia de la Constitución de Cádiz en Cuba, de 1812 a 1814, cuando Fernando VII regresó de su exilio dorado en Francia e impuso a sangre y fuego la monarquía abso-

${ }^{7}$ Este texto constitucional también es conocido con el nombre popular de "la Pepa", por el hecho fortuito de haberse proclamado el 19 de marzo de 1812, día consagrado por el santoral católico a San José. 
luta. En Cuba, estos sucesos pasaron casi inadvertidos, y al explicarnos esa extraña situación, no encontramos otra respuesta que el cuidado que puso Fernando VII en no molestar en demasía a los terratenientes y poderosos plantadores cubanos, que no solo tenían poder económico, sino también poder político, aunque fuera de manera soterrada. En muchos casos, los hacendados criollos eran más ricos que muchos rancios aristócratas españoles, que tenían muchos títulos y mucho abolengo, pero poco oro en sus bolsas. No convenía causar muchos disgustos a los hombres ricos de la única posesión que España conservaba en América. Recuérdese que para esta fecha, la mayor parte de los movimientos independentistas americanos habían triunfado, con las consiguientes pérdidas millonarias para la corona española y la mancha para el orgullo ibérico.

En esta primera etapa, que va de 1814 a 1820, conocida como la "Edad Dorada" del reformismo, mientras España se ocupaba de intentar la reconquista de sus colonias perdidas, la isla gozó de una bonanza económica, por supuesto, para los grandes propietarios y explotadores esclavistas; ni pensar que para el pueblo haya habido mejoras económicas y mucho menos para la población esclava, que sostuvo sobre sus espaldas tal desarro1lo. Sin embargo, fue un marco propicio para que los autores cubanos, que en esa época luchaban para establecer un pensamiento político, filosófico y jurídico genuinamente nacional, tuvieran amplio margen para desplegar sus concepciones.

Corresponde mencionar en primerísimo lugar al padre Félix Varela, que por esos años llevó a cabo la primera y más importante reforma de la enseñanza filosófica en Cuba. Cuando cinco o seis años más tarde se verificó en España la segunda proclamación de la Constitución de Cádiz, con la aprobación, al menos formal, del rey, el padre Varela, impulsado por el obispo Espada, que fue el principal promotor de esta Ilustración cubana, ${ }^{8}$ amparado en su carácter de obispo y en el poder que tenía la Iglesia católica, se dio a la tarea de fundar la primera cátedra de Derecho constitucional (Derecho político se nombraba en esa época) que existió en Hispanoamérica. Esta fundación se basa en una disposición del gobierno liberal español en el llamado Trienio Liberal, ${ }^{9}$ por la que

${ }^{8}$ Juan José Díaz de Espada y Fernández de Landa era el nombre completo de este obispo español, que desde su consagración en la catedral habanera se dedicó a realizar acciones encaminadas a favorecer la cultura, la educación, el saneamiento y la moralización de la diócesis. Fue un intelectual de proyecciones clarísimas, con una preocupación constante por formar hombres de bien con amplios conocimientos. El obispo Espada estuvo acompañado de nombres tan ilustres en el ámbito intelectual cubano como Tomás Romay, José Agustín Caballero y el propio Félix Varela, entre otros.

${ }^{9}$ Periodo de la historia española entre 1820 y 1823 , cuyo nombre responde al paréntesis libe- 
se mandó que en cada ciudad española y en cada ciudad de los territorios ultramarinos se constituyera una plaza de la constitución y se fundara una cátedra de estudios constitucionales. El padre Varela se presentó al concurso de oposición y recibió en propiedad la docencia de la cátedra, que comenzó a impartir a principios de 1821. Todos los testimonios de la época coinciden en que se trató de un hecho inaudito y de un impacto extraordinario; personas colgadas de las ventanas hacían todo lo posible por entrar al salón de conferencias, completamente colmado. La mayoría escuchaba desde fuera, sin poder ver. Las conferencias dictadas por Varela habían sido un rotundo éxito. La formación religiosa permeó el iusnaturalismo de esos estudios de Derecho constitucional, y se afirmaba la posesión de derechos imprescriptibles, de los que ninguna nación podía hacer traslado o remoción, por ser superiores a la ley. Uno de los valores esenciales que movió a los mambises cubanos a plantearse el complejo problema de la constitución fue el de la igualdad de los hombres, sin distinciones de ninguna clase. Sobre este aspecto Varela decía:

El hombre libre que vive en una sociedad justa, no obedece sino a la ley;

ral y constitucionalista que abrió el rey absolutista Fernando VII. Es también conocido como Trienio Constitucional. mandarle invocando otro nombre es valerse de uno de los muchos prestigios de la tiranía, que solo producen su efecto en almas débiles. El hombre no manda a otro hombre; la ley los manda a todos [...] Uno de los resultados de la verdadera libertad es el derecho de igualdad, que quiere decir el derecho de que se aprecien sus perfecciones y méritos del mismo modo que otros iguales que se hayan en cualquier individuo, de manera que una acción no pierde por la persona que la ejecuta. ${ }^{10}$

Al valorar los resultados fenomenales que habían tenido las clases, el obispo Espada ordenó la publicación por primera vez en Cuba (y hasta donde se tiene noticia, en Hispanoamérica) del primer tratado de Derecho constitucional. Antes, habían circulado traducciones de Rousseau, de Montesquieu, etc. Ese primer tratado de Derecho constitucional escrito en español contenía las lecciones impartidas por el padre Varela y llevó el título de Observaciones sobre la Constitución Política de la Monarquía española. El libro solo fue reeditado en Cuba en 1944, y desde entonces esperamos por un editor osado y de finísimo olfato que nos regale esta maravillosa y aleccionadora lectura, que tiene un lugar cimero,

\footnotetext{
${ }^{10}$ Jorge Ibarra Cuesta, Varela el precursor. Un estudio de época, 2008, La Habana, Editorial de Ciencias Sociales, p. 61.
} 
aunque no muy conocido, entre los clásicos del pensamiento cubano.

A donde vamos llegando ya en nuestras ideas, es a que todo ese periodo de intensos debates, potenciados por la libertad de prensa, la igualdad jurídica y las concepciones individualistas de los principios de libertad, igualdad y propiedad, preceptos todos derivados de la letra de la Constitución de Cádiz, propició en Cuba la publicación de gran cantidad de periódicos, panfletos y folletos que defendían los ideales constitucionales y republicanos, la libertad, los derechos individuales, las garantías judiciales, etc. No hay duda de que gran parte de ese ímpetu constitucionalista se debe a la obra del padre Varela, pues pasaron por su aula José de la Luz y Caballero, José Antonio Saco, Nicolás Escobedo, Domingo del Monte, José María Heredia y una lista larga. Cada uno de estos hombres contribuyó, dentro de su ámbito de acción y de sus posibilidades reales, a conformar un ideal de nación cubana, pero de nación republicana, no monárquica; una nación de respeto y garantías para los derechos individuales. Varela, derivado de su éxito en las clases, fue electo diputado a las Cortes de Madrid, que luego se mudarían otra vez a Cádiz ante el avance de las tropas francesas que venían a reponer en el trono a Fernando VII, ${ }^{11}$

\footnotetext{
${ }^{11}$ Tras la celebración del Congreso de Verona (octubre-noviembre de 1822), los países miembros de la Santa Alianza: Francia, Rusia, Austria y Prusia,
}

y aunque no pudo regresar a Cuba, en suelo patrio quedaron sus principales discípulos. José de la Luz y Caballero dirigió la cátedra de Filosofía hasta que fue cerrada y luego fundó su propia escuela, gastando su dinero y minando su salud, donde tuvo entre sus discípulos a hombres de la talla de Perucho Figueredo, Ignacio Agramonte, Julio Sanguily, Antonio Zambrana, entre muchos otros. Años después, estos alumnos recordaban las palabras del ya viejo pedagogo: "para que Cuba sea libre, yo soy maestro de escuela". Muchos años más tarde, José Martí rememoraba que no había un hogar en Tampa y en Cayo Hueso donde no estuviera colgada, junto a la bandera cubana, una foto de Don Pepe, como cariñosamente llamaban los cubanos al maestro José de la Luz y Caballero.

El padre Varela sabía del alcance de sus prédicas; ya en el prólogo de su famoso Cartas a Elpidio anunciaba:

No creo haber ofendido a ninguna persona determinada, pero no ha

acordaron el envío de tropas para devolver el régimen absolutista en España. Esta invasión fue conocida como los Cien Mil Hijos de San Luis, en alusión al discurso del soberano francés Luis XVIII en el que invocó al "Dios de San Luis" y a la ayuda segura de "cien mil franceses". Estas tropas, mandadas por Luis Antonio de Borbón, duque de Angulema, cumplieron su objetivo sin encontrar apenas resistencia, y apoyados por una masa realista que ansiaba la vuelta absolutista, instalaron nuevamente a Fernando VII en el trono. 
sido posible prescindir de dar algunos palos a ciertas clases. Quisiera que hubieran sido más flojos; pero estoy hecho a dar de recio, y se me va la mano $[\ldots]$ Preveo que este avechucho puede acarrearme algunos enemigos, pero ya es familia a cuyo trato me he habituado, pues hace tiempo que estoy como el yunque, siempre bajo el martillo. ${ }^{12}$

Para concluir, consideramos que no es aventurado decir que la obra filosófica, jurídica y política de Varela, Saco y José de la Luz, hasta donde lo permitieron las circunstancias, constituyó la raíz de ese ideal republicano y constitucionalista que llevó a nuestros mambises a luchar por una Carta Magna en la manigua insurrecta. Estos hombres pueden ser llamados perfectamente padres del constitucionalismo cubano, padres de la idea una Cuba

${ }^{12}$ Félix Varela, Cartas a Elpidio sobre la impiedad, la superstición y el fanatismo en sus relaciones con la sociedad, 1836, Madrid, Imprenta de D. León Amarita, pp. 4-5. libre e independiente. Para terminar, nada mejor que las palabras martianas sobre aquel alumbramiento de Guáimaro:

¡Con qué cuidado debe andar la pluma, y con qué ternura, cuando se escribe sobre aquellos hombres! Otros andamos por la senda abierta: ¡ellos fueron los que abrieron la senda! Por donde quiera que andemos los de ahora, hemos de andar con el sombrero quitado. Lengua, todos tenemos; pero espadas, pocos. De lo más bello del mundo es aquella juventud imperiosa, que no quería república patricia ni historia a medias; y aquel patriciado que sentó canas con la juventud. El desinterés es lo más bello de la vida; y el interés es su fealdad. El día de la generosidad absoluta en la historia de Cuba, ;fue el día 10 de abril!! ${ }^{13}$
${ }^{13}$ José Martí, La Revolución de 1868, 1968, La Habana, Instituto del Libro, selección y prólogo de Julio Le Riverend, p. 344. 